\title{
Efficient autotransporter-mediated extracellular secretion of a heterologous recombinant protein by Escherichia coli
}

lan Henderson ( $\square$ i.henderson@uq.edu.au )

University of Queensland

Irene Beriotto

University of Birmingham

Christopher Icke

University of Queensland

Yanina Sevastsyanovich

University of Birmingham

Amanda Rossiter

University of Birmingham

Giacomo Romagnoli

GlaxoSmithKline (Italy)

Silvana Savino

GlaxoSmithKline (Italy)

Jeffrey Cole

University of Birmingham

Allan Saul

GSK Vaccines Institute for Global Health

Calman MacLennan

University of Oxford

Adam Cunningham

University of Birmingham

Francesca Micoli

GSK Vaccines Institute for Global Health

\section{Research Article}

Keywords: Autotransporter, Escherichia coli, Recombinant protein production, Secretion, Inclusion bodies, Pet autotransporter platform

Posted Date: February 3rd, 2022 
DOI: https://doi.org/10.21203/rs.3.rs-1300960/v1

License: (c) (1) This work is licensed under a Creative Commons Attribution 4.0 International License. Read Full License 


\section{Abstract}

Background: The autotransporter protein secretion system has previously been used to target the secretion of heterologous proteins to the bacterial cell surface and the extracellular milieu at laboratory scale. The platform is of particular interest for the production of "difficult" recombinant proteins that might cause toxic effects when produced intracellularly. One such protein is IrmA. IrmA is a vaccine candidate that is produced in inclusion bodies requiring refolding. Here, we describe the use and scale-up of the autotransporter system for the secretion of an industrially relevant protein (IrmA).

Results: A plasmid expressing irmA was constructed such that the autotransporter platform could secrete IrmA into the culture supernatant fraction. The autotransporter platform was suitable for the production and purification of IrmA with comparable physical properties to the protein produced in the cytoplasm. The production of IrmA was translated to scale-up protein production conditions resulting in a yield of $29.3 \mathrm{mg} / \mathrm{L}$ of IrmA from the culture supernatant.

Conclusions: The autotransporter platform is a viable method for the production, secretion, and purification of a "difficult" to produce protein on an industrially relevant scale. Use of the autotransporter platform could reduce the number of downstream processing operations required thus accelerating the development time and reducing costs for recombinant protein production.

\section{Background}

Recombinant protein production is a cornerstone of the molecular biology revolution and the biotechnology industry. Escherichia coli is frequently used to produce proteins [1]. However, many of these are produced in the cytoplasm of $E$. coli and often aggregate into inclusion bodies, requiring multiple downstream processing steps to achieve a soluble, pure, functional protein, and thereby increasing production costs and time to market. In contrast, accumulation of the target protein in the culture supernatant fraction could reduce downstream processing steps and production time, resulting in significant cost savings [2]. Many bacterial secretion systems have been harnessed to deliver recombinant heterologous proteins into the culture supernatant. One such system that has been well studied, which is attractive because of its simplicity, is the classical autotransporter (AT) secretion system. This system is found widely among most Gram-negative bacterial species [3-5]. These AT proteins are a subset of the Type 5 secretion system (T5SS) and typically include an N-terminal signal sequence that mediates translocation across the cytoplasmic membrane, a functionally discrete passenger domain, an autochaperone (AC) domain that assists in folding of the passenger domain, and a $\mathrm{C}$-terminal $\beta$-barrel domain that facilitates secretion of the passenger domain across the bacterial outer membrane [5-7]. By replacing the passenger domain with a protein of interest, the AT system can be engineered such that the protein of interest can be displayed on the bacterial cell surface or released into the extracellular milieu [2]. 
The AT platform offers an alternative to other traditional systems for cytoplasmic expression and may offer a solution to produce "difficult proteins" that are toxic or misfolded when retained within the cell. Particularly attractive is that the protein is secreted extracellularly. Extracellular secretion could enable

toxic protein production to be maintained by bacteria for longer than traditional systems. Additionally, protein can accumulate in the medium at high concentrations. However, while the AT platform can accumulate heterologous proteins in the culture medium at laboratory scale, there is no evidence to support the utility of the AT platform for recombinant protein production at scales useful for industrial protein production. Consequently, we sought to produce interleukin receptor mimic protein A (IrmA) to test the efficacy of the AT platform for the production of heterologous protein in an industrial setting.

IrmA (16.9 kDa) is an interleukin receptor mimic produced by uropathogenic Escherichia coli (UPEC), a causative agent of urinary tract infections (UTIs) [8]. UTIs affect over half of all women during their lifetime with the associated antimicrobial treatment accounting for $15 \%$ of outpatient prescriptions [9]. IrmA is a putative vaccine candidate that when used in immunization studies can induce protection after challenge with UPEC [10]. Currently, IrmA for use as a vaccine is produced in E. coli in inclusion bodies and is purified from the cytoplasmic fraction using a pre-established proprietary methodology that includes refolding of the protein, affinity chromatography, sample desalting, ion exchange chromatography and finally a dialysis step (we have designated this form of the protein IrmAC).

Previously we have investigated the use of the Pet AT as a platform for the secretion of heterologous proteins. Pet is a member of the serine protease ATs of the Enterobacteriaceae (SPATEs) as such it is autocatalytic. This autocatalytic activity liberates the passenger domain from the AT enabling direct secretion into the extracellular environment [2]. Here we investigated whether the Pet AT platform could be used to produce and secrete IrmA into the culture medium (IrmA-S) at an industrial scale, thus reducing the complexity of its production process, saving time, and costs.

\section{Results}

Production of secreted IrmA-S. Previously, we described an AT protein production platform based on the Pet serine protease of E. coli. We demonstrated that the Pet AT platform could secrete a variety of heterologous proteins to the bacterial cell surface or into the extracellular milieu [2].IrmA was selected to test whether the Pet AT platform could be exploited to produce a "difficult" protein with commercial relevance. The irmA open reading frame without a stop codon was cloned into the Novagen vector, pET22b, containing the pet AT sequence. The irmA gene sequence was cloned into the $N c o l$ and $S b f l$ sites enabling fusion at the $\mathrm{C}$-terminus with the $\mathrm{Pet} A C$ domain and at the $\mathrm{N}$-terminus with a polyhistidine tag preceded by the PelB signal sequence (Figure 1A). The whole Pet passenger domain to the autochaperone (AC) domain was replaced with the irmA gene sequence. The final construct was termed pET22b-IrmA-S (Figure S1). The resulting plasmid encodes the fusion of Pet AT and IrmA under control of an IPTG inducible T7 promoter. As the fusion protein is secreted the PelB signal sequence is removed by the Sec pathway during translocation (Figure 1B). Then, the $\beta$-barrel is inserted into the outer membrane 
by the BAM complex, IrmA and fused AC are secreted via the $\beta$-barrel and cleaved at the Passenger domain cleavage site in the a-helix. This retains a $14.2 \mathrm{kDa}$ section of the Pet AC domain fused to the Cterminus of IrmA. IrmA-S contains a $1.4 \mathrm{kDa}$ six histidine tag and linker, $14.2 \mathrm{kDa}$ of IrmA (native IrmA without the Sec signal sequence), and $14.2 \mathrm{kDa}$ Pet AC domain. The final molecular mass of IrmA-S is $29.8 \mathrm{kDa}$.

The cysteine residues present in the antigen IrmA are separated by 60 residues but disulphide-bonded loops of over 20 residues were shown to be incompatible with the Pet AT translocation though the $\beta$ barrel domain [11]. Considering this, the plasmid pET22b-IrmA-S was transformed into the host strain $E$. coli BL21*DE3 dsbA::aph, a strain lacking DsbA which is required for cysteine bond formation in the bacterial periplasm, and protein expression was induced with IPTG during growth in lysogeny broth. Proteins secreted into the extracellular milieu were precipitated by the addition of trichloroacetic acid and analysed by SDS-PAGE separation followed by Coomassie staining or Western blotting using anti-His tag antibodies (Figure 1C and 1D, respectively). Coomassie staining revealed a single 35-kDa band was in the culture fluid. Western blotting confirmed that this band contained a His tag. Therefore, this band was presumed to be IrmA-S despite the difference in expected mass, $29.8 \mathrm{kDa}$, and the observed mass, approximately $35 \mathrm{kDa}$, after separation by SDS-PAGE. The suspected IrmA-S band was excised and digested by incubation with trypsin. The resulting peptides were analysed by liquid chromatography tandem mass spectrometry and peptides corresponding to both the Pet AT domain and IrmA were identified (Table S1). Furthermore, the identity of IrmA-S was confirmed using liquid chromatography tandem mass spectrometry without trypsin digestion. The measured molecular mass of IrmA-S was 29849.46 Da. This was consistent with the predicted theoretical molecular mass of IrmA-S of 29841 Da (Figure S2). Additionally, IrmA-S accounted for more than $90 \%$ of the total protein present within the culture medium (Figure 1C). Thus, IrmA-S was secreted via the Pet AT platform and constituted the majority of the proteins present in the extracellular milieu.

Purification of secreted IrmA. Having verified that the Pet AT platform could be used to produce IrmA-S as a secreted protein, we sought to determine a method to purify the protein to homogeneity. As IrmA-S was a substantial component of the culture supernatant we predicted that a single purification step would be adequate for protein purification to homogeneity. Accordingly, an E. coli BL21*DE3 dsbA::aph strain harbouring the $p E T 22 b$-IrmA-S plasmid was grown in 2 L of LB. Cells were harvested by centrifugation and the culture medium containing IrmA-S was isolated. The culture supernatant fraction was concentrated by membrane ultrafiltration and dialysed against the binding buffer. The concentrated culture medium was incubated with cobalt magnetic beads and subsequently IrmA-S was eluted with imidazole. Fractions from the purification were analysed by SDS-PAGE (Figure 2). A band corresponding to IrmA-S was detected in the eluted fractions but not in the flow-through fractions or washes. Therefore, IrmA-S was successfully purified from the extracellular milieu in a single purification step.

Characterisation of secreted IImA. Purified IrmA-S was compared with IrmA-C to check that the two proteins produced with different systems were biochemically similar. IrmA-S and IrmA-C were first analysed by SDS-PAGE in reducing and non-reducing conditions (Figure 3A). Most of IrmA-S and IrmA-C 
migrated as a single protein band when samples were reduced and boiled. There were two bands in the unreduced IrmA-S sample. Approximately $85 \%$ of the non-reduced and unboiled IrmA-S migrated faster than the reduced and boiled IrmA-S, which is consistent with the reduction of the IrmA disulphide bridge in the reduced and boiled samples [8]. Despite the size difference both IrmA-S and IrmA-C migrated as monomers in the presence of SDS.

The T5SS passenger domain is usually translocated through its cognate $\beta$-barrel domain in an unfolded structure [6]. The passenger domain folds after translocation and it has been proposed that its folding could be the driving force for the translocation [12]. Passenger domain folding has been shown to happen only after translocation [13]. The fold of IrmA-S was compared to that of IrmA-C by differential scanning calorimetry (Figure 3B). A transition was registered at a melting temperature of $56.1^{\circ} \mathrm{C}$ for IrmA$\mathrm{C}$ and $52.8^{\circ} \mathrm{C}$ for IrmA-S. This transition was the transition of the $\operatorname{IrmA}$ domain that the two proteins share. A second transition at $68.4^{\circ} \mathrm{C}$ was detected for IrmA-S likely related to the presence of 115 amino acids of the Pet autochaperone domain which is absent in IrmA-C. A biphasic transition for proteins containing two domains has been previously observed [14-16]. The similarity of the IrmA-C and lower IrmA-S transition temperatures confirms that both share the IrmA protein structure. Furthermore, IrmA-S and IrmA-C were analysed by reverse phase chromatography. IrmA-S and IrmA-C were eluted at the same retention time suggesting that the two proteins had a similar hydrophobicity (Figure 3C). Therefore, despite the differences in production and purification method of IrmA-S and IrmA-C, both display similar biophysical properties.

Clone selection for IrmA-S production. Having demonstrated that IrmA-S had a comparable conformation to IrmA-C, we tested a scaled-up production process to determine whether the Pet AT platform was compatible with the high yields required for industrial scale recombinant protein production. High cell density cultures are used to produce a higher amount of the protein. Fermentation is routinely used to achieve high cell density cultivation of E. coli [17]. A nutrient-rich medium was chosen instead of standard LB to support growth at high cell density for the fermentation production of IrmA-S. In addition, bacteria were grown at a lower temperature, $25^{\circ} \mathrm{C}$ instead of 30 to $37^{\circ} \mathrm{C}$, to lower the cellular stress that occurs when an exogenous protein is overexpressed.

As there could be variations between transformants, the pET22b-IrmA-S plasmid was transformed into the E. coli $\mathrm{BL} 21^{*} \mathrm{DE} 3 d s b A:: a p h$ and 8 clones were screened for IrmA-S accumulation. Three were selected for further analysis: Clones 2, 3, and 7. These clones were grown in $50 \mathrm{~mL}$ of complex medium. IrmA-S production was induced with $0.5 \mathrm{mM}$ IPTG. Growth of the bacteria was assessed by measuring culture $\mathrm{OD}_{590}$ (Figure $4 \mathrm{~A}$ ). Clone 2 grew more rapidly than clones 3 and 7 , which were similar. Samples of culture medium were taken every hour until 5 hours post-induction and again 24 hours post-induction. Proteins in the culture medium were precipitated by trichloroacetic acid, separated by SDS-PAGE and stained with Coomassie (Figure 4B) or subjected to immunoblotting with anti-His antibodies (Figure 4C). SDS-PAGE analysis revealed that protein impurities were present in the culture medium at all time points analysed. This phenomenon was not observed when IrmA-S was produced from small scale LB cultures (Figure 1C 
and 1D). Clone 2 was shown to have fastest growth and comparable IrmA-S production at earlier time points (Figure 4C). For this reason, it was chosen for further study.

Scale-up of IrmA production. Having demonstrated that IrmA-S could be produced in high density cultures, we next sought to investigate production in scaled-up conditions. A complex medium was used to support bacterial growth to high cell density. A $4 \mathrm{~L}$ culture was grown at a fixed temperature of $25^{\circ} \mathrm{C}$. IrmA-S production was induced with $0.5 \mathrm{mM}$ IPTG for $3 \mathrm{~h}$. Cells were harvested by centrifugation and the culture medium was isolated. Due to the presence of process impurities in the culture medium (Figure 4B) several downstream processing steps were included. A scheme of the process used is shown in Figure $5 \mathrm{~A}$. The culture medium was concentrated by tangential flow filtration to reduce the sample volume and remove impurities. The use of a membrane with a $10 \mathrm{kDa}$ cut off allowed the isolation of IrmA-S in the retentate. Subsequently, the concentrated culture medium was applied to a $\mathrm{Ni}^{2+}$ affinity column. After washing IrmA-S was eluted with imidazole. Imidazole was removed from the eluted fractions by buffer exchange chromatography using a HiPrep Desalting Column. The resulting IrmA-S enriched fraction was applied to a Q Sepharose column. After washing the antigen was eluted with $\mathrm{NaCl}$. The purified fraction was separated by SDS-PAGE and analysed by Coomassie staining (Figure 5B) or immunoblotting (Figure 5C). Samples were retained from each stage of the process so that the yield of IrmA-S could be assessed (Table S2). After $3 \mathrm{~h}$ of induction the amount of IrmA-S produced in the culture medium was $29.3 \mathrm{mg} / \mathrm{L}$ (Table 1). Despite the further purification steps required for the batch fermentation compared to the small-scale purification a high amount of IrmA-S was isolated. Therefore, the Pet AT platform is suitable for batch fermentation resulting in high yields of secreted soluble protein.

\section{Discussion}

Many new biopharmaceuticals have been approved by FDA, including hormones, growth factors, antibodies, vaccines, fusion proteins and therapeutic enzymes [18]. Three hundred and forty-seven biopharmaceuticals had been approved by July of 2019, 94 of which were produced in E. coli [1]. Sales figures for these drugs are growing, which in turn is increasing competition due to the advent of the first biosimilars on the market. Upstream and downstream processing procedures contribute significantly to the final price of the drug itself and it has been estimated that the cost of downstream processing alone could account for up to $80 \%$ of the total cost of the final drug manufacture. It has therefore become clear that new systems for effective, low-cost recombinant protein production are required and a need for simplification in such processes has been emphasised [18]. The Pet AT platform was proposed as a possible method to achieve these goals [2].

The standardisation, optimisation, and reproducibility of protein production is a crucial issue in the attempt to produce biopharmaceuticals at a lower cost using bacterial systems. Typically, heterologous proteins are produced in the cytoplasm of the bacterial cell. Cytoplasmic protein production can result in the formation of inclusion bodies or a reduction in biomass due to the production of a toxic protein. Proteins in inclusion bodies must be refolded and may not fold into their native structure. Additionally, the cytoplasmic content of cells must be released by lysis, which may affect protein fold and result in 
product contamination. Furthermore, the desired protein must now be isolated from the lysate potentially resulting in contamination by autologous proteins requiring further purification steps and equipment. These steps are costly, laborious, and each additional step results in product loss. Thus, a possible way to increase production efficiency of an exogenous protein is by targeting the protein to the culture medium in which purification of the protein would be more cost-effective. Currently, IrmA is purified from bacterial inclusion bodies. Thus, the production process is not cost effective as it requires refolding. Here, the Pet AT platform is an advantageous alternative. The results obtained for the small-scale production of IrmA-S with the Pet AT platform were promising. IrmA-S accounted for more than $90 \%$ of the culture supernatant sample (Figure 1C). IrmA-S present in the supernatant was soluble and did not require a refolding step. Thus, the purification of IrmA-S in a single step directly from the culture supernatant is possible.

Prior to this study, the possibility to scale up a production process based on the Pet AT platform through fermentation had not been explored. Having verified the ability of the Pet AT platform to produce IrmA-S at the laboratory scale, we wanted to demonstrate that the system could be scaled up to obtain high protein yields. We demonstrated that the Pet AT platform could be scaled up and was compatible with culturing conditions such as batch fermentation. A yield of $29.3 \mathrm{mg} / \mathrm{L}$ of IrmA-S in the culture supernatant is very promising. This small-scale study demonstrated that production of a "difficult" protein using the Pet AT platform was feasible. Optimization of the fermentation process to increase yields and decrease contaminants has the potential to result in a highly cost-efficient process.

\section{Conclusions}

In conclusion, we describe a system that can secrete a soluble heterologous protein during batch fermentation culture. The Pet AT platform is a versatile single protein system that could avoid the typical issues of cytoplasmic recombinant protein production at the industrial scale, enabling simple and efficient protein purification from the culture supernatant of fermentation cultures.

\section{Methods}

\section{Bacterial strains and growth conditions}

E. coli $\mathrm{DH} 5 \mathrm{a}$ and E. coli $\mathrm{BL} 21 * \mathrm{DE} 3$ were purchased from Invitrogen and Novagen, respectively. $E$. coli $\mathrm{DH} 5 \mathrm{a}$ was used for cloning and plasmid preparation. E. coli BL21*DE3 dsbA::aph was constructed by $\mathrm{P} 1$ transduction from E. coli TOP10 dsbA::aph [11]. E. coli BL21*DE3 dsbA::aph was routinely cultured in Lysogeny Broth (LB), $10 \mathrm{~g} / \mathrm{L}$ tryptone, $5 \mathrm{~g} / \mathrm{L}$ yeast extract, and $5 \mathrm{~g} / \mathrm{L} \mathrm{NaCl}$, and used for recombinant protein production. For batch fermentation $E$. coli $\mathrm{BL} 21 * \mathrm{DE} 3 d s b A$ :: aph was cultured in complex media composed of $45 \mathrm{~g} / \mathrm{L}$ yeast extract, $10 \mathrm{~g} / \mathrm{L}$ glycerol, $16 \mathrm{~g} / \mathrm{L} \mathrm{K}_{2} \mathrm{PO}_{4}$ and $4 \mathrm{~g} / \mathrm{L} \mathrm{KH_{2 }} \mathrm{PO}_{4}$ in scaled-up conditions. If required, culture media were supplemented with the appropriate antibiotics and inducers as required: ampicillin $(100 \mu \mathrm{g} / \mathrm{mL})$, kanamycin $(25 \mu \mathrm{g} / \mathrm{mL})$ and IPTG $(0.5 \mathrm{mM})$. 


\section{SDS-PAGE and Immunoblotting}

Sample Buffer Laemmli $2 X$ concentrate (Sigma) and $2 \mu \mathrm{L}$ of DTT $1 \mathrm{M}$ (Invitrogen) were mixed with protein samples prior to incubation at $100^{\circ} \mathrm{C}$ for 5 minutes. When a non-reducing and non-denaturing analysis was required, samples were treated as specified before but omitting DTT and heating. Samples were loaded onto a pre-casted 4-12\% SDS-PAGE protein gel (Invitrogen) with PageRuler Plus Prestained Protein Ladder (Thermo Scientific). Proteins were detected using Brilliant Blue G - Colloidal concentrate, Electrophoresis Reagent for protein detection (Sigma) as per the manufacturer's instructions. Specific proteins were detected by immunoblotting. In brief, proteins were separated on an SDS-PAGE gel and transferred to nitrocellulose using the iBlot Gel Transfer System (Life Technologies). The membrane was incubated with a Blotting Solution ( $5 \% \mathrm{w} / \mathrm{v}$ skimmed milk powder and PBS supplemented with $0.1 \% \mathrm{v} / \mathrm{v}$ Tween 20). The membrane was washed three times with PBST (PBS supplemented with $0.1 \% \mathrm{v} / \mathrm{v}$ Tween 20 ) and incubated with a dilution of the appropriate primary antibody in Blotting Solution. A 1:5,000 dilution of a mouse monoclonal antibody raised against poly-histidine tag was used. The membrane was washed three times with PBST prior to incubation with a dilution of the appropriate secondary antibody in Blotting Solution. A 1:30.000 dilution of anti-mouse alkaline phosphatase-conjugated antibodies (Sigma) was used as secondary antibodies. The membrane was washed three times with PBST prior to protein detection with BCIP/NBT-Purple Liquid Substrate System for Membranes (Thermo Fisher) or the Opti-4CN Substrate Kit (BIORAD) according to manufacturer's instructions.

\section{Culture supernatant protein precipitation}

Culture volumes were adjusted according to the $\mathrm{OD}_{590}$ of the cultures. Cells were removed by centrifugation at $3724 \mathrm{x} \mathrm{g}$ for $20 \mathrm{~min}$ at $4^{\circ} \mathrm{C}$. Culture supernatants were filtered through $0.22 \mu \mathrm{m}$ filters (Millipore) and a 1:10 dilution v/v of ice-cold 100\% trichloroacetic acid was added to each sample. Samples were incubated on ice for $45 \mathrm{~min}$ and precipitated proteins were spun down at $15,366 \times \mathrm{g}$ for 45 min at $4^{\circ} \mathrm{C}$. Supernatants were discarded and pellets were resuspended in $1 \mathrm{~mL}$ of ice-cold $100 \%$ acetone. Samples were centrifuged at $18,407 \times g$ for 1 hourat $4^{\circ} \mathrm{C}$ to remove acetone. The resulting pellets were resuspended in $100 \mu \mathrm{L}$ of Sample Buffer Laemmli $2 X$ concentrate (Sigma) supplemented with saturated Tris-solution and 1 M DTT (Invitrogen) prior to analysis by SDS-PAGE and immunoblotting.

\section{Small scale purification of IImA}

Cultures were grown at temperatures from 30 to $37^{\circ} \mathrm{C}$ for small scale IrmA-S production. An aliquot of $150 \mathrm{~mL}$ of culture supernatant was concentrated to $3-4 \mathrm{~mL}$ using VivaSpin Columns (Sartorius) with 10 $\mathrm{kDa}$ cut-off. The concentrated culture supernatant was dialysed overnight at $4^{\circ} \mathrm{C}$ against Binding Buffer (50 mM Na${ }_{2} \mathrm{HPO}_{4} \mathrm{pH} \mathrm{8,300} \mathrm{mM} \mathrm{NaCl}$, and 0.01\% Tween 20). Samples were incubated overnight with 150 $\mu \mathrm{L}$ of Dynabeads (Life Technologies) at $4^{\circ} \mathrm{C}$. In the morning, Dynabeads were washed 4 times with $1 \mathrm{~mL}$ of Binding Buffer and then six times with $100 \mu \mathrm{L}$ of Elution Buffer $(50 \mathrm{mM} \mathrm{Na} 2 \mathrm{HPO} 4 \mathrm{pH} 8,300 \mathrm{mM} \mathrm{NaCl}$, $0.01 \%$ Tween 20 , and $300 \mathrm{mM}$ Imidazole).

\section{Batch Fermentation}


A $50 \mathrm{~mL}$ culture of the selected strain was inoculated from glycerol stock. The medium used was composed of $45 \mathrm{~g} / \mathrm{L}$ yeast extract, $10 \mathrm{~g} / \mathrm{L}$ glycerol, $16 \mathrm{~g} / \mathrm{L} \mathrm{K}_{2} \mathrm{PO}_{4}$ and $4 \mathrm{~g} / \mathrm{L} \mathrm{KH}_{2} \mathrm{PO}_{4}$ supplemented with $100 \mu \mathrm{g} / \mathrm{mL}$ ampicillin. A $100 \mathrm{~mL}$ of broth was inoculated to an $\mathrm{OD}_{590}$ of 0.2 with an overnight aerated culture and grown in a shaking incubator at $25^{\circ} \mathrm{C}$ at $180 \mathrm{rpm}$. A 7 litres autoclavable ADI Bioreactor (Applikon) with a working volume of 4 litres containing the medium specified above was inoculated to an $\mathrm{OD}_{590}$ of 0.05 . Foam formation was prevented with PPG at a concentration of $0.75 \mathrm{~mL} / \mathrm{L}$, temperature was set at $25^{\circ} \mathrm{C}$, $\mathrm{pH}$ was maintained at 7.2 with $4 \mathrm{M} \mathrm{NaOH}$ and $2 \mathrm{M} \mathrm{H}_{3} \mathrm{PO}_{4}$, dissolved oxygen concentration, and stirrer speed were controlled with the Bioxpert software (Applikon). The fermenter culture was induced at $\mathrm{OD}_{600} 12.5$ for $3 \mathrm{~h}$ with $0.5 \mathrm{mM}$ IPTG. Simultaneously, $30 \mathrm{~g} / \mathrm{L}$ glycerol, $0.25 \mathrm{~g} / \mathrm{L}$ $\mathrm{MgSO}_{4}$ and ampicillin $50 \mathrm{mg} / \mathrm{L}$ were added. Bacteria were pelleted by centrifugation at $12,000 \times \mathrm{g}$ for $1 \mathrm{~h}$ at $4^{\circ} \mathrm{C}$ to remove cell debris and particulates. Subsequently, the supernatant fraction was filter sterilized through a $0.22 \mu \mathrm{m}$ filtering unit (Corning) and kept at $4^{\circ} \mathrm{C}$ prior to further analysis.

\section{Scaled-up purification of IrmA}

Two litres of fermentation supernatant were concentrated 20 -fold at $4^{\circ} \mathrm{C}$ by tangential flow filtration using a Sartocon Slice 200 (200 $\mathrm{cm}^{2}$ filter area, 10-kDa cut-off, hydrosart membrane, Sartorius). The retentate containing IrmA-S was then diafiltrated against 20 volumes of PBS. During the diafiltration step, the retentate volume was kept constant, the input pressure was maintained at 1.8-2.0 bar and transmembrane pressure at 1.1-1.2 bar. The retentate was collected for further purification of IrmA-S.

The tangential flow filtration retentate was purified by affinity chromatography using an ÄKTA Purifier 10 (GE, Healthcare) and a $1 \mathrm{~mL}$ HisTrap HP Column (GE Healthcare). Protein absorbance at 214, 260 and $280 \mathrm{~nm}$ was monitored. The mobile phase (Binding buffer $\mathrm{HP}$ ) was composed of $50 \mathrm{mM} \mathrm{Na}_{2} \mathrm{HPO}_{4} \mathrm{pH}^{8}$, and $300 \mathrm{mM} \mathrm{NaCl}$ and was used to equilibrate the column with a $1 \mathrm{~mL} / \mathrm{min}$ flow rate in 10 column volumes. The culture supernatant was loaded onto the column at $1 \mathrm{~mL} / \mathrm{min}$ flow rate. The column was washed with 5 column volumes of Binding buffer HP. The IrmA-S was eluted with Elution buffer HP (50 $\mathrm{mM} \mathrm{Na}_{2} \mathrm{HPO}_{4} \mathrm{pH} 8,300 \mathrm{mM} \mathrm{NaCl}$, and $400 \mathrm{mM}$ imidazole) at $1 \mathrm{~mL} / \mathrm{min}$ flow rate in 10 column volumes. One $\mathrm{mL}$ fractions were collected at every purification step for further analysis. If required, the fractions containing IrmA-S were pooled and dialysed against $2 \mathrm{~L}$ of Binding Buffer HP overnight at $4^{\circ} \mathrm{C}$. Proteins were quantified by the Micro BCA Assay using the Micro BCA Protein Assay Kit (Thermo Scientific).Affinity chromatography fractions containing the antigen IrmA-S were combined. Salt was removed by buffer exchange chromatography. A Sephadex G-25 column HiPrep 26/10 Desalting (GE Healthcare) was equilibrated with $50 \mathrm{mM}$ Tris- $\mathrm{HCl} \mathrm{pH} 8.0$ at a $4 \mathrm{~mL} / \mathrm{min}$ flow rate. The sample was loaded on the column and eluted isocratically at a $4 \mathrm{~mL} / \mathrm{min}$ flow rate for 1.5 column volumes. Fractions of the void volume were pooled and subjected to further purification by ion exchange chromatography. An HiTrap Q Sepharose FF column (GE Healthcare) was used. Protein absorbance at 215, 260 and $280 \mathrm{~nm}$ was detected. The column was equilibrated with $50 \mathrm{mM}$ Tris- $\mathrm{HCl}, \mathrm{pH} 8.0$ (Binding buffer) at a $1 \mathrm{~mL} / \mathrm{min}$ flow rate. Fractions were loaded on the column with a $1 \mathrm{~mL} / \mathrm{min}$ flow rate. The column was washed with 5 column volumes of Binding buffer. IrmA-S was eluted with a linear gradient from 0 to $100 \%$ of $\mathrm{NaCl} 500$ 
$\mathrm{mM}$ in 20 column volumes of Binding buffer. One $\mathrm{mL}$ fractions were collected and analysed by SDSPAGE to determine the fractions containing IrmA-S.

\section{Differential scanning calorimetry}

A MicroCal VP-Capillary DSC instrument (GE Healthcare) with integrated autosampler was used for Differential scanning calorimetry (DSC). In brief, DSC scans were in the temperature range of $10-130^{\circ} \mathrm{C}$ with a thermal ramping of $150^{\circ} \mathrm{C}$ per $\mathrm{h}$ and a $5 \mathrm{~s}$ filter period. Samples were exchanged in PBS $\mathrm{pH}$ 7.4. For each sample $500 \mu \mathrm{L}$ at a concentration of $12 \mu \mathrm{M}$ (estimated by Nanodrop) were analysed; the samples were transferred to a 96-well plate and left in the instrument autosampler at $5^{\circ} \mathrm{C}$ until analysis. A $30 \mu \mathrm{M}$ lysozyme solution was analysed at the beginning and at the end of the set of experiments to confirm a high degree of reproducibility of the data collected $(T m=71.2 \pm 0.1)$. Data were analysed using the Origin 7 software (OriginLab), subtracting the data recorded for a sample containing only buffer to the reference data.

\section{Reverse-phase high pressure liquid chromatography}

IrmA-C and IrmA-S were analysed by reverse-phase high pressure liquid chromatography (RP-HPLC) with a UPLC system Acquity-UPLC (Waters). Samples were analysed on a BEH C4 Column $300 \AA$ (2,1 x 150 $\mathrm{mm}, 1,7 \mu \mathrm{m}$ particles size, Acuity UPLC Protein). The mobile phase was composed by buffer $\mathrm{A}(0.1 \% \mathrm{TFA})$ and buffer $B(0.1 \%$ TFA, $90 \%$ acetonitrile). Samples were eluted at a flow rate of $4 \mathrm{~mL} / \mathrm{min}$ in three steps: with $97.7 \%$ buffer $A$ and $2.3 \%$ buffer $B$ for 2.5 min; with $0 \%$ buffer $A$ and $100 \%$ buffer $B$ for 10 min; and finally with $97.7 \%$ buffer $A$ and $2.3 \%$ buffer $B$ for $2.5 \mathrm{~min}$, at $0.4 \mathrm{~mL} / \mathrm{min}$ flow rate. Protein absorbance at 215,260 and $280 \mathrm{~nm}$ was measured.

\section{Declarations}

\section{Ethics approval and consent to participate}

Not applicable.

\section{Consent for publication}

Not applicable.

\section{Availability of data and materials}

All data generated or analysed during this study are included in this published article.

\section{Funding}

This work was funded by the European Union FP7-PEOPLE-2012-ITN VADER project (Grant agreement ID: 316940). 
Authors' contributions - provide individual author contribution

I.H., A.S., A.C. and C.M. conceived the project. I.B. did the experiments. J.C., F.M., G.R., S.S., A.E., and Y.S. supervised the lab research. I.B., C.I. and I.H. analysed the data. C.I. and I.B. wrote the manuscript. All authors edited the manuscript.

\section{Acknowledgements}

We would like to thank Paolo Costantino for his help in this work.

\section{References}

1. Gierach I, Galiardi JM, Marshall B, Wood DW. Protein drug production and formulation. Remington. 2021;23:489-547.

2. Sevastsyanovich YR, Leyton DL, Wells TJ, Wardius CA, Tveen-Jensen K, Morris FC, et al. A generalised module for the selective extracellular accumulation of recombinant proteins. Microb Cell Fact. 2012;11:69.

3. Desvaux M, Khan A, Beatson SA, Scott-Tucker A, Henderson IR. Protein secretion systems in Fusobacterium nucleatum: Genomic identification of Type 4 piliation and complete Type $V$ pathways brings new insight into mechanisms of pathogenesis. Biochim Biophys Acta. 2005;1713:92-112.

4. Henderson IR, Lam AC. Polymorphic proteins of Chlamydiaspp.-autotransporters beyond the Proteobacteria. Trends Microbiol. 2001;9:573-8.

5. Desvaux M, Parham NJ, Henderson IR. Type V protein secretion: Simplicity gone awry? Curr Issues Mol Biol. 2004;6:111-24.

6. Henderson IR, Navarro-Garcia F, Desvaux MM, Fernandez RC, Ala'Aldeen D, Ala'Aldeen D. Type V Protein Secretion Pathway: the Autotransporter Story. Microbiol Mol Biol Rev. 2004;68:692-744.

7. Desvaux M, Parham NJ, Scott-Tucker A, Henderson IR. The general secretory pathway: A general misnomer? Trends Microbiol. 2004;12:306-9.

8. Moriel DG, Heras B, Paxman JJ, Lo AW, Tan L, Sullivan MJ, et al. Molecular and structural characterization of a novel Escherichia coli interleukin receptor mimic protein. MBio. 2016;7.

9. Dielubanza EJ, Schaeffer AJ. Urinary tract infections in women. Med Clin North Am; 2011;95:27-41.

10. Moriel DG, Bertoldi I, Spagnuolo A, Marchi S, Rosini R, Nesta B, et al. Identification of protective and broadly conserved vaccine antigens from the genome of extraintestinal pathogenic Escherichia coli. Proc Natl Acad Sci U S A. 2010;107:9072-7.

11. Leyton DL, Sevastsyanovich YR, Browning DF, Rossiter AE, Wells TJ, Fitzpatrick RE, et al. Size and conformation limits to secretion of disulfide-bonded loops in autotransporter proteins. J Biol Chem. 2011;286:42283-91.

12. Leyton DL, Rossiter AE, Henderson IR. From self sufficiency to dependence: Mechanisms and factors important for autotransporter biogenesis. Nat Rev Microbiol. 2012;10:213-25. 
13. Junker M, Schuster CC, McDonnell A V., Sorg KA, Finn MC, Berger B, et al. Pertactin $\beta$-helix folding mechanism suggests common themes for the secretion and folding of autotransporter proteins. Proc Natl Acad Sci U S A. 2006;103:4918-23.

14. Catanzano F, Gambuti A, Graziano G, Barone G. Interaction with D-glucose and thermal denaturation of yeast hexokinase B: A DSC study. J Biochem. 1997;121:568-77.

15. Koide S, Bu Z, Risal D, Pham TN, Nakagawa T, Tamura A, et al. Multistep denaturation of Borrelia burgdorferi OspA, a protein containing a single-layer $\beta$-sheet. Biochemistry. 1999;38:4757-67.

16. Kumar DP, Tiwari A, Bhat R. Effect of pH on the stability and structure of yeast hexokinase A. Acidic amino acid residues in the cleft region are critical for the opening and the closing of the structure. $J$ Biol Chem. 2004; 279:32093-9.

17. Korz DJ, Rinas U, Hellmuth K, Sanders EA, Deckwer WD. Simple fed-batch technique for high cell density cultivation of Escherichia coli. J Biotechnol. 1995;39:59-65.

18. Walsh G. Biopharmaceutical benchmarks 2010. Nat Biotechnol. 2010;32:917-24.

\section{Tables}

Table 1: Accumulation of IrmA-S in the culture supernatant

\begin{tabular}{ll} 
Time post induction & Amount of IrmA-S secreted into the culture supernatant $(\mathrm{mg} / \mathrm{L})$ \\
\hline $1 \mathrm{~h}$ & 6.38 \\
\hline $2 \mathrm{~h}$ & 16.61 \\
\hline $3 \mathrm{~h}$ & 29.25 \\
\hline
\end{tabular}

\section{Figures}


A

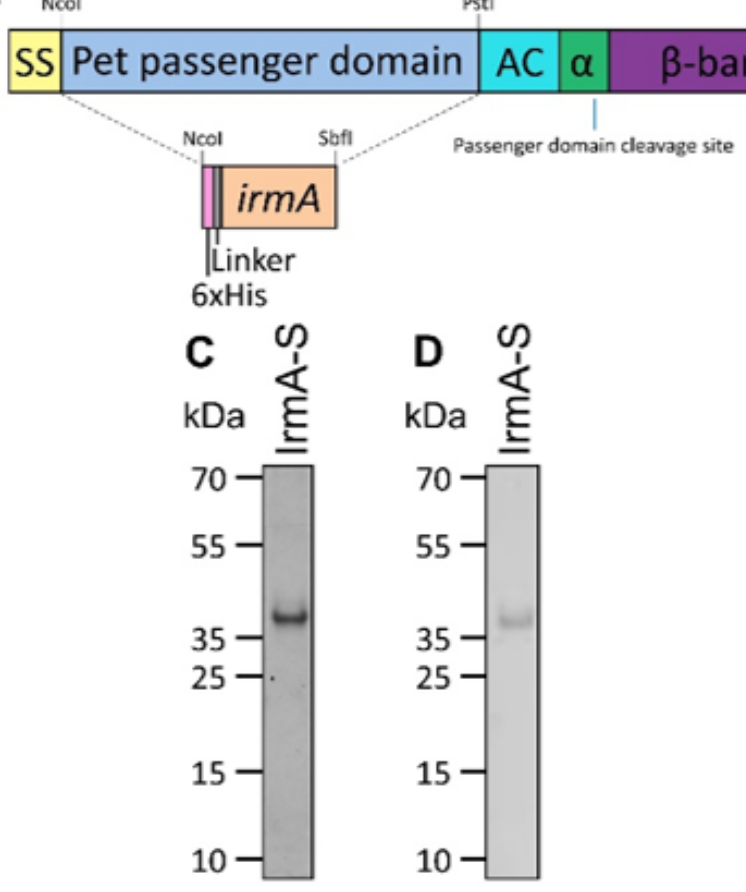

Coomassie stain $\alpha-H i s$ tag

B

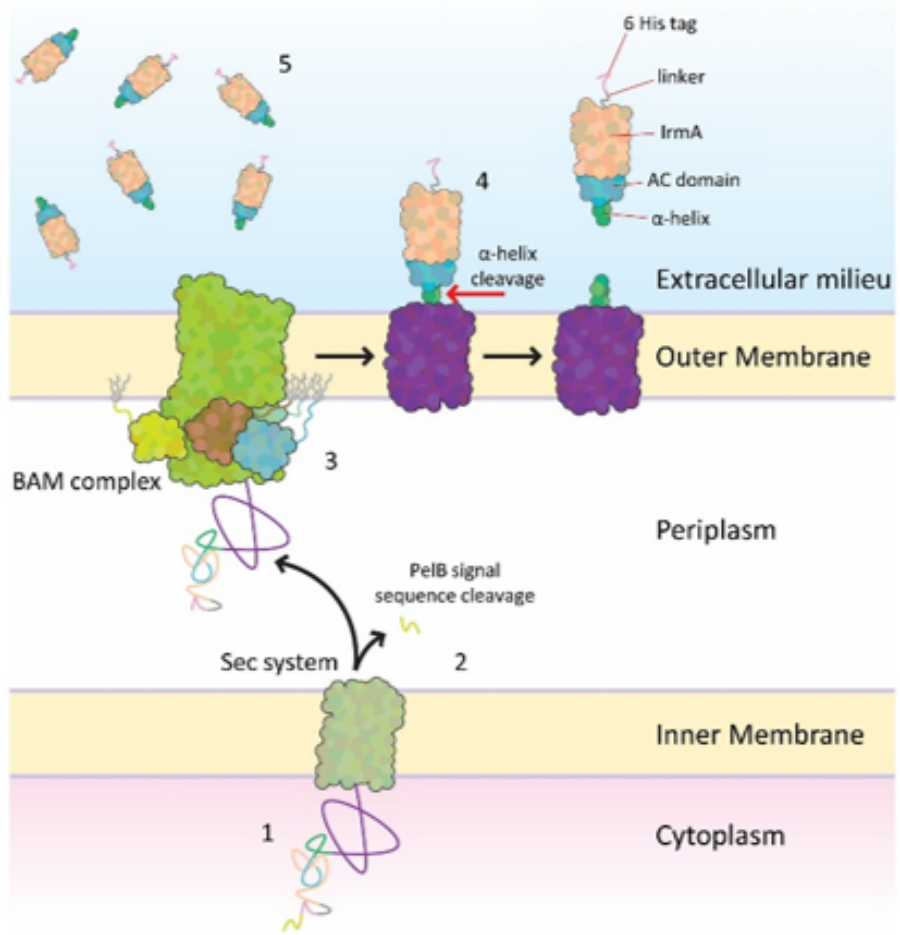

\section{Figure 1}

IrmA-S construct and secretion from E. coli BL21. (A) Schematic diagram of the IrmA-S construct, not to scale. The irmA gene is inserted into the Pet AT platform replacing the native Pet passenger domain leaving a pelB signal sequence (SS), a $6 \mathrm{x}$ His tag, a linker region, the irmA gene, the autochaperone domain (AC), and the a-helix (a) the site at which the passenger domain is cleaved from the $\beta$-barrel. The plasmid encoding this construct was termed pET22b-IrmA-S. (B) Secretion of IrmA-S by the AT platform occurs in 5 steps: 1 . The AT platform is produced in the cytoplasm. 2. The AT platform is secreted into the periplasm by the Sec system and the PelB signal sequence is cleaved. 3. The AT platform is inserted into the outer membrane by the BAM complex. 4. The folded AT platform undergoes autoproteolysis releasing IrmA-S. 5. IrmA-S accumulates in the culture medium. (C) The culture supernatant of E. coli BL21*DE3 $d s b A$ :: aph harbouring the plasmid pET22b-IrmA-S after IPTG induction was analysed for the presence of IrmA-S by SDS-PAGE. (D) The same sample was subjected to immunoblotting using a-His antibodies. Note that although the theoretical molecular mass of IrmA-S is $29.8 \mathrm{kDa}$, it migrated in the gel with a higher apparent molecular mass. 


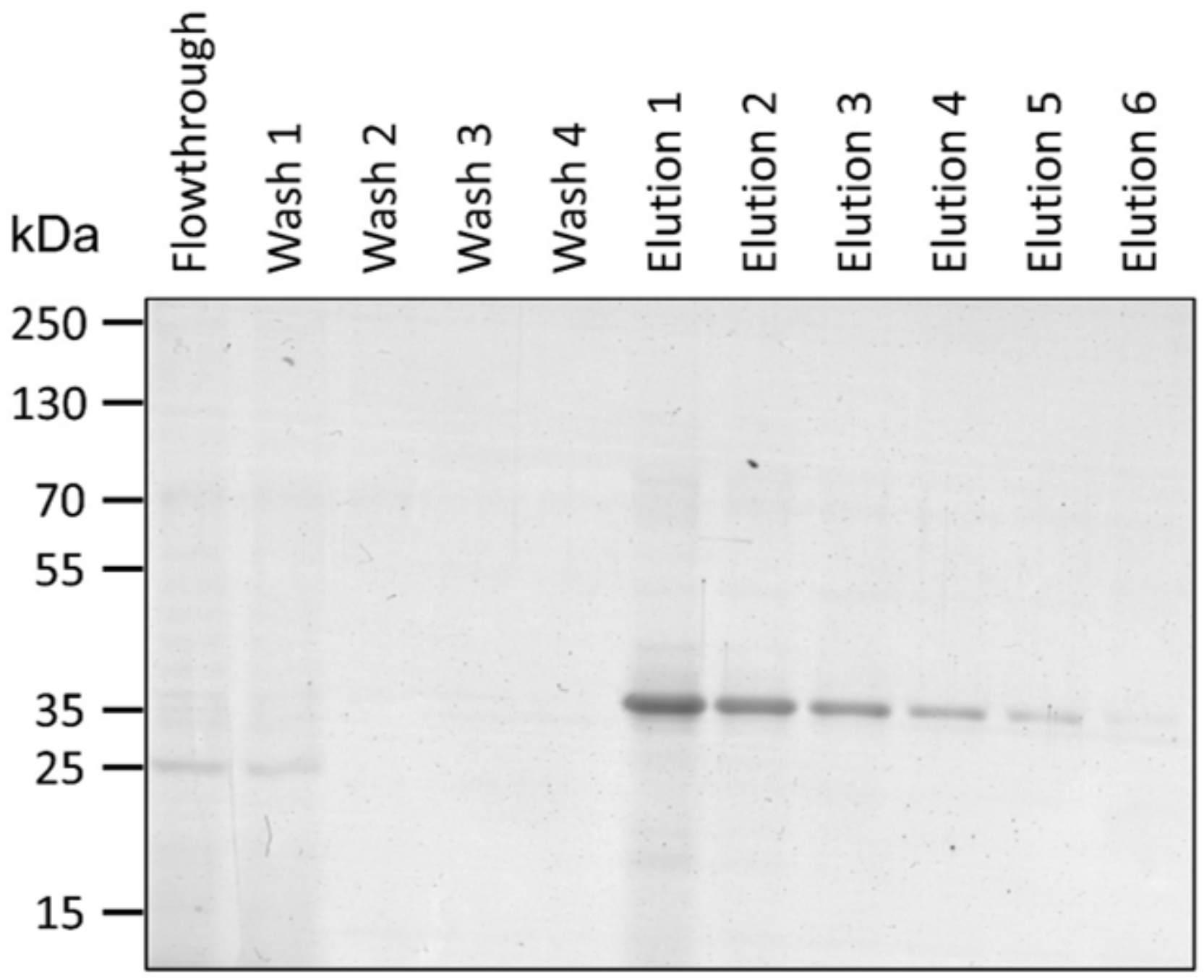

Figure 2

Purification of IrmA-S from the culture supernatant. The culture medium of E. coli BL21*DE3 $d s b A:: a p h$ harbouring the plasmid pET22b-IrmA-S was harvested. The culture medium was concentrated by membrane ultrafiltration and dialysed against the binding buffer prior to incubation with the magnetic beads for purification. IrmA-S was eluted with imidazole. 

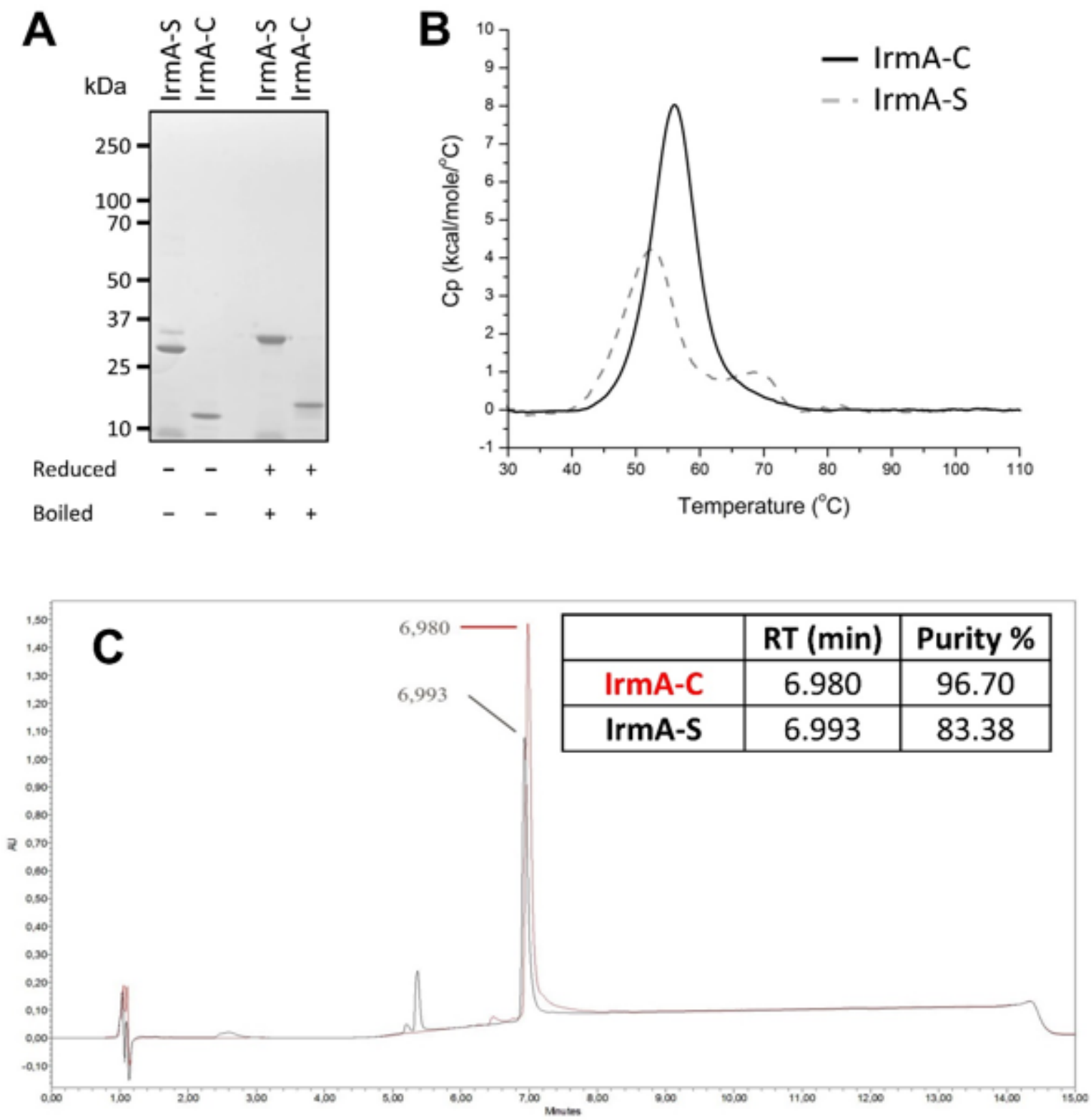

Figure 3

Comparison of IrmA-C and IrmA-S. (A) IrmA-S and IrmA-C were analysed by SDS-PAGE with or without heating and reduction by DTT. (B) IrmA-S and IrmA-C were analysed by differential scanning calorimetry to investigate their folding and thermal stability. Scans were recorded and the spectra are reported in solid line for IrmA-C and dashed line for IrmA-S. (C) IrmA-S and IrmA-C were analysed by reverse-phase chromatography. 
A

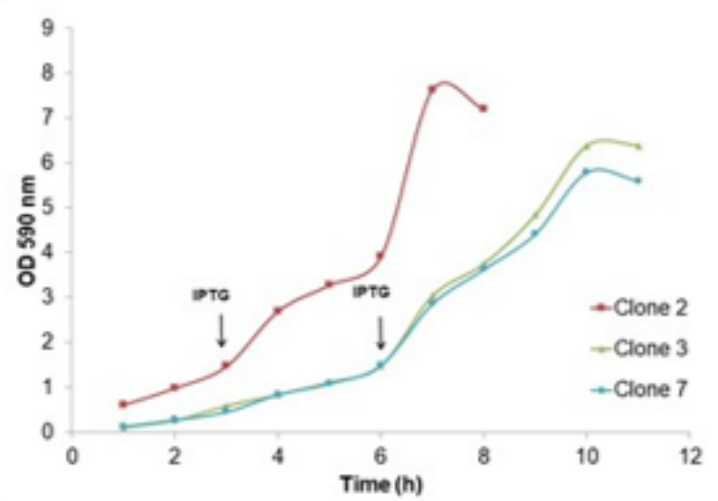

B
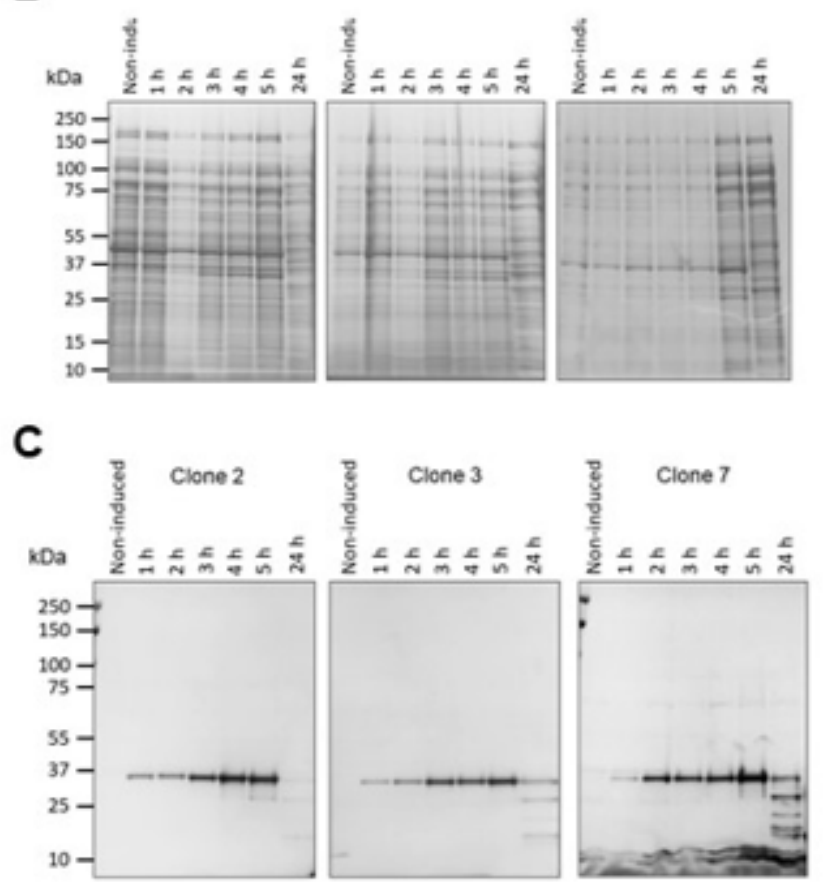

Figure 4

Screening of clones for IrmA-S production. Bacterial clones 2, 3 and 7 were grown in of $50 \mathrm{~mL}$ complex medium. IrmA-S expression was induced with IPTG after culture reached an $\mathrm{OD}_{590}$ of 1 . (A) The growth of the clones was monitored with $\mathrm{OD}_{590}$ measurements. For 5 hours after IPTG induction the culture medium for each clone was harvested and the proteins were precipitated in presence of trichloroacetic acid. Precipitated protein samples were separated by SDS-PAGE and stained with Coomassie (B) or analysed by immunoblotting with a a-His antibody (C). 
A

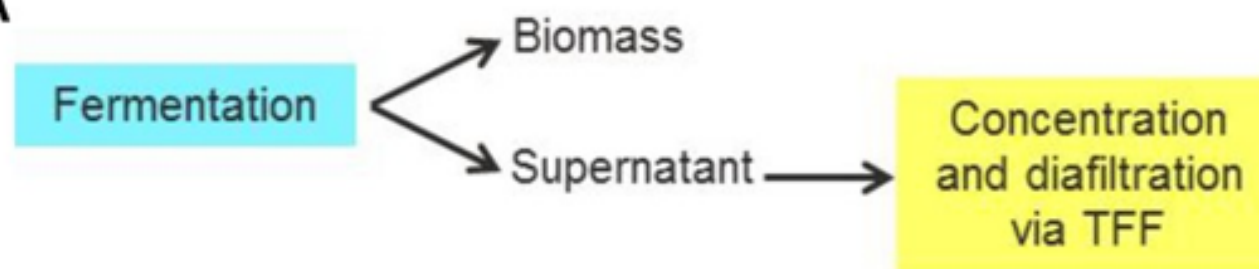

B
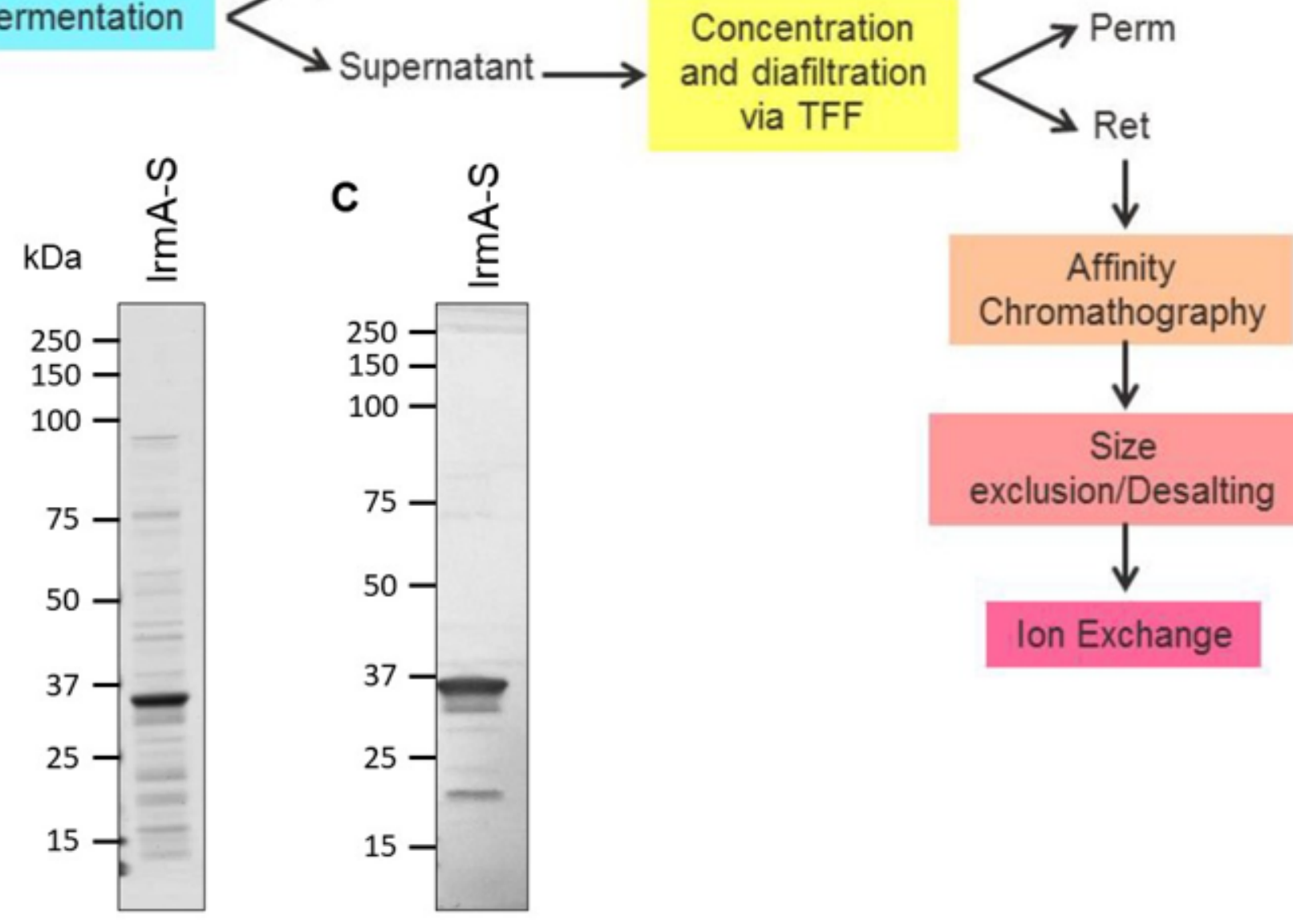

\section{Figure 5}

Scaled-up production process for IrmA-S. (A) A schematic of the production process for IrmA-S. After fermentation of the isolated clone, the culture medium was separated and concentrated via tangential flow filtration (TFF). The concentrated culture medium was purified by $\mathrm{Ni}^{2+}$ affinity chromatography. The sample was desalted by size exclusion chromatography to remove the imidazole in the eluted fractions. Finally, the antigen was purified by ion exchange chromatography. A culture of clone 2 was grown in a batch fermenter. IrmA-S accumulation in the culture medium was induced for 3 hours with IPTG. The culture supernatant was isolated and concentrated 20 -fold by tangential flow filtration. The IrmA-S was purified from the concentrated culture medium by affinity chromatography and ion exchange chromatography. The purified antigen was analysed by SDS-PAGE and stained with Coomassie (B) or analysed by immunoblotting with a-His antibody (C).

\section{Supplementary Files}

This is a list of supplementary files associated with this preprint. Click to download. 
- Supplementaryfiguresandtables.docx

Page 19/19 